\title{
A Generic Database Indexing Framework for Large-Scale Geographic Knowledge Graphs
}

\author{
Yuhan Sun \\ Arizona State University, Tempe AZ \\ ysun138@asu.edu
}

\begin{abstract}
The paper proposes Riso-Tree, a generic indexing framework for geographic knowledge graphs. Riso-Tree enables fast execution of graph queries that involve spatial predicates (aka. GraSp). The proposed framework augments the classic R-Tree structure with pre-materialized sub-graph entries. Riso-Tree first partitions the graph into sub-graphs based on their connectivity to the spatial sub-regions. The proposed index allows for fast execution of GraSp queries by efficiently pruning the traversed vertexes/edges based upon the materialized sub-graph information. The experiments show that the proposed Riso-Tree achieves up to two orders magnitude faster execution time than its counterparts when executing GraSp queries on real knowledge graphs (e.g., WikiData).
\end{abstract}

\section{CCS CONCEPTS}

- Information systems $\rightarrow$ Query optimization; Geographic information systems; Wikis; Hierarchical data models;

\section{KEYWORDS}

Spatial index, GeoSpatial Knowledge Graph, range query

\section{ACM Reference Format:}

Yuhan Sun and Mohamed Sarwat. 2018. A Generic Database Indexing Framework for Large-Scale Geographic Knowledge Graphs. In Proceedings of 26th ACM SIGSPATIAL International Conference on Advances in Geographic Information Systems (SIGSPATIAL '18). ACM, New York, NY, USA, 10 pages. https://doi.org/10.1145/3274895.3274966

\section{INTRODUCTION}

With the ubiquity of spatial data, vertexes or edges in knowledge graphs can possess spatial location attributes side by side with other non-spatial attributes. For instance, as of June 2018 the Wikidata knowledge graph contains 48, 547, 142 data items (i.e., vertexes) to date and $\approx 13 \%$ of them have spatial location attributes [21]. Figure 1a depicts another example; a knowledge graph (extracted from Yelp) where a vertex represents either a place (restaurant or movie theater) or a person. An edge between a person $p$ and a place $v$ exists if $p$ visited $v$ before and an edge between two persons means they are friends. Figure $1 \mathrm{~b}$ shows another knowledge graph that models the electricity utility network as a graph. There are power stations, power towers and electricity customers (including factories and

Permission to make digital or hard copies of part or all of this work for personal or classroom use is granted without fee provided that copies are not made or distributed for profit or commercial advantage and that copies bear this notice and the full citation on the first page. Copyrights for third-party components of this work must be honored.

For all other uses, contact the owner/author(s).

SIGSPATIAL '18, November 6-9, 2018, Seattle, WA, USA

(C) 2018 Copyright held by the owner/author(s).

ACM ISBN 978-1-4503-5889-7/18/11.

https://doi.org/10.1145/3274895.3274966

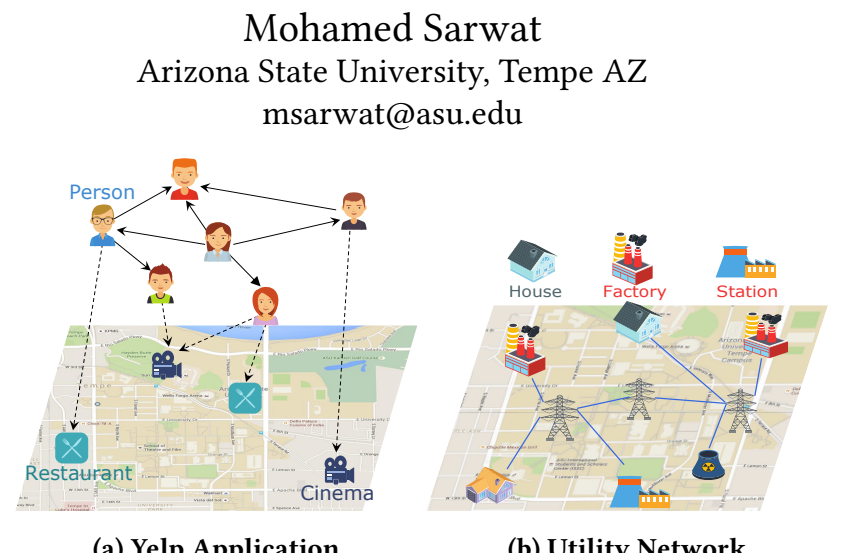

(a) Yelp Application

(b) Utility Network

houses). Each graph vertex possesses a spatial attribute, which represents its location within the city. Edges in the utility graph show how these spatial entities are connected topologically. Furthermore, many of the existing knowledge graphs (e.g., Wikidata) consist of real-world entities and facts, which naturally possess spatial attributes (aka. Geographic Knowledge Graph).

A graph database system such as Neo4j [12] and Titan [20] provides an efficient way for managing and accessing graph data. Several graph database systems already support spatial attributes. In the Yelp example, the application developer may use Neo4j to create a vertex for a place (e.g., restaurant) and add a spatial location attribute to it. In the utility network knowledge graph example, the application developer may use a graph database system to create a vertex for each power station and assign spatial location attribute (polygon representing the geographical boundaries of the station). The co-existence of graph and spatial data in the same geographic knowledge graph allows users to issue queries like:

- Q1:'Find all places in Seattle that are co-visited by at least two friends or friends of friends'

- Q2:'Find single-family houses in Tempe, AZ that receive power from a distribution site connected to the central power station in Maricopa County'.

In the rest of the paper, we refer to such type of Graph queries with Spatial predicates as GraSp queries. Note that GraSp is not a new query type. In fact, there has been a flurry of recent research papers that tackle a variety of GraSp applications $[1,2,5,6,11$, $15,17,19]$. For instance, the research work about Range Friends and Nearest Friends queries find friends of a user within a given distance and the user's top- $K$ nearest friends, respectively [1]. The utility network application is addressed in [2] (e.g., Q2). Other GraSp applications include spatial entity resolution and discovering related spatial entities. Such applications motivate the development of a generic approach that efficiently processes a GraSp query 
posed against a graph database. A main challenge is how a graph database system (GDBMS) can efficiently evaluate spatial predicates (e.g., range, K-Nearest Neighbors, spatial join) in a graph query. Existing GDBMS optimizers follow one of the following strategies to evaluate GraSp:

GraphTraverse first traverses the graph to find the matched graph patterns and then evaluates the spatial predicates. Then, the vertexes that cannot satisfy the spatial predicate (e.g., places within the extents of Seattle in Q1) are filtered out. Although GraphTraverse answers GraSp queries correctly, it may possibly visit many unnecessary vertexes/edges during the graph traversal. Another approach, namely SpaIndex, initially builds a spatial index [3, 9, 14], e.g., R-Tree, over the spatial vertexes in the graph. The query processor in SpaIndex runs in two steps: (1) Step I applies the index to find all spatial vertexes that can satisfy the spatial predicate; (2) Step II then traverses the graph starting from the set of spatial vertexes. Step I may retrieve spatial vertexes that satisfy the spatial predicate but do not match the query graph; That will unnecessarily traverse graph paths. In conclusion, both approaches exhibit unacceptable performance in applications that need to query the geographic knowledge graph in real-time or near real-time.

In this paper, we propose Riso-Tree, an index that efficiently answers Graph queries with Spatial predicates (GraSp). Unlike

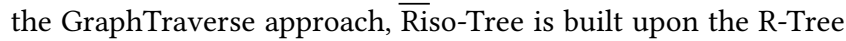
spatial index structure to prune sub-graphs that are guaranteed not to satisfy the spatial predicate. Unlike the SpaIndex approach that prunes objects solely based on spatial predicates, Riso-Tree prunes branches that are guaranteed not to result in a matching in the graph. The closest approach (let's call it GeoEncoding) to Riso-Tree adds spatial predicate support into an existing SPARQL query engine [10]. The main differences between GeoEncoding and Riso-Tree are: (1) GeoEncoding was primarily designed to execute SPARQL queries over RDF data, whereas Riso-Tree is natively designed to support generic graph queries processed by de-facto graph database systems. (2) GeoEncoding implements a spatial encoding technique that allows the RDF query optimizer to effectively select either the SpaIndex or GraphTraverse approach to prune spatial predicates. Riso-Tree goes one step further by selectively materializing graph patterns within each spatial region in the R-tree structure, and then leverages the materialized patterns to re-write a more selective graph query. The GDBMS can then optimize and process the re-written query faster than SpaIndex and GraphTraverse approaches. That said, we believe that a GDBMS can utilize both GeoEncoding and Riso-Tree together to run GraSp queries even faster. In summary, the contributions of this paper are as follows:

- The paper proposes Riso-Tree, a generic index for geographic knowledge graphs. Riso-Tree augments the classic R-Tree structure with sub-graph entries (Section 3.1). Riso-Tree partitions the graph into sub-graphs based on their connectivity to the spatial sub-regions. The index allows for fast execution of GraSp queries by efficiently pruning the traversed vertexes/edges based upon the sub-graph information. The paper also describes how to efficiently construct and maintain Riso-Tree in a graph database (Section 3.2).

- The paper describes three query processing algorithms that leverage the proposed Riso-Tree index structure to evaluate generalpurpose spatial predicates such as spatial range, join and K-Nearest

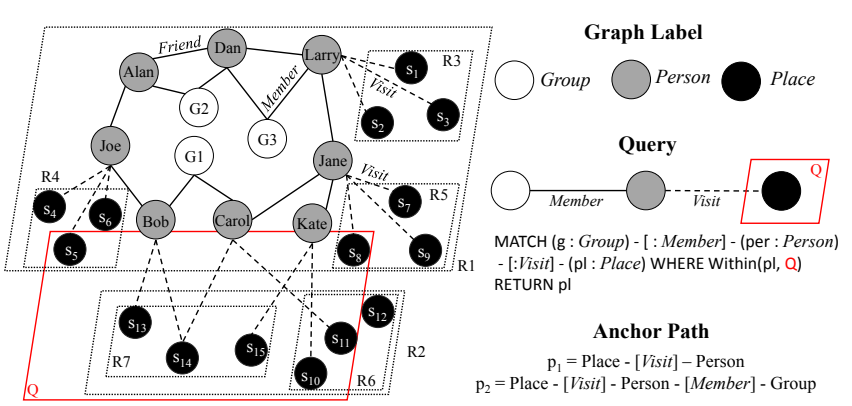

Figure 2: Running Example

Neighbors (KNN) in a graph query (Sections 4). The paper also explains how a graph query processor integrates and optimizes such algorithms with other graph query operators, and eventually executes the input GraSp query.

- The paper performs a comprehensive experimental evaluation based on the actual implementation of the Riso-Tree in Neo4j (open source graph database system) (Section 5). The experiments show that the proposed Riso-Tree achieves up to two orders magnitude faster execution time than its counterparts when executing GraSp queries on real spatial knowledge graphs and geosocial graph dataset (Foursquare, Gowalla) as well as synthetic datasets.

\section{PRELIMINARIES}

Geographic Knowledge Graph Model: The paper assumes that the knowledge graph is stored in and managed by a graph database system. Hence, we consider a property / labeled graph $G=$ $(V, E, \varphi, \psi)$ such that (1) $V$ is a set of vertexes, (2) $E$ is a set of edges, (3) $\varphi$ is a mapping function $\varphi: V \rightarrow \mathcal{L}_{V}$, where $\mathcal{L}_{V}$ is a set of vertexes labels or types, (4) $\psi$ is a mapping function $\psi: E \rightarrow \mathcal{L}_{E}$, where $\mathcal{L}_{E}$ is a set of edge labels or types [18]. A geographic knowledge graph is a property graph that possesses at least one spatial label/type in $\mathcal{L}_{V}$. In such a graph, some vertexes semantically represent spatial objects, which possess spatial location attributes, e.g., point, polygon. The spatial attribute of a vertex is denoted as v.loc. Figure 2 shows a geographic knowledge graph with three types of vertexes, Group, Person and Place, depicted with white, gray and black respectively. Spatial vertexes (Place) are denoted with $s_{i}$ where $i=1,2, \ldots, 15$. Each spatial vertex has a location in the 2-D space. There are three types of edges, Member, Friend and Visit. A Member edge means a person is a member of a group. A Friend edge means the two persons are friends. A Visit edge means a place is visited by a person.

Graph Query: Given a graph $G=(V, E, \varphi, \psi)$, a graph query $G_{q}=\left(V_{q}, E_{q}, \varphi_{q}, \psi_{q}\right)$ finds all the maps $f$ from $G_{q}$ to $G$ such that: (1) $\forall v \in V_{q}, \varphi_{q}(v)=\varphi(f(v))$; and (2) $\forall(u, v) \in E_{q}, \psi_{q}(u, v)=$ $\psi(f(u), f(v))$. A graph query with spatial predicate (abbr. GraSp) is a normal graph query except that it has spatial predicates specified on some query vertexes. A GraSp query can be denoted as $\left\langle G_{q}, S P_{q}\right\rangle$ where $G_{q}=\left(V_{q}, E_{q}, \varphi_{q}, \psi_{q}\right)$ and $S P_{q}$ is a set of spatial predicates. An example of a GraSp query is depicted in Figure 2. The $G_{q}$ component of the query is Group-[Member $]-$ Person - [Visit $]-$ Place. The predicate is $S P_{q}=\{(\mathrm{Place}, Q)\}$ where $Q$ is the spatial range 


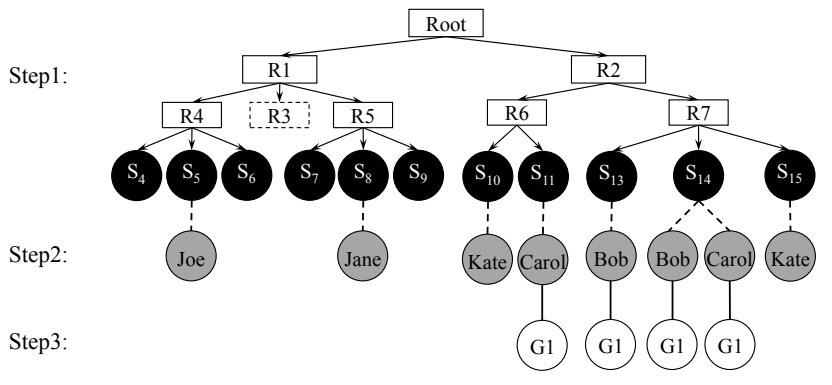

(a) SpaIndex

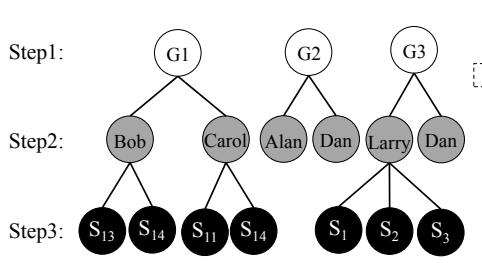

(b) GraphTraverse

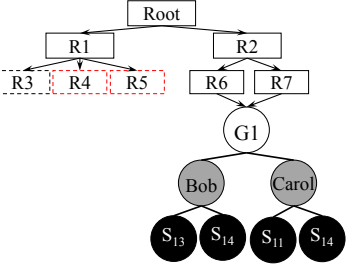

(c) Riso-Tree

Figure 3: Figure 3a depicts the steps the SpaIndex approach takes to process the query given in Figure 2: Step 1: Search R-Tree to filter out the objects that are not located within the region $Q . R 3$ is not accessed because it does not overlap with $Q$. Step 2: Traverse the graph from each spatial object to obtain persons that visit such place. Step 3: Search the graph for all groups that each person is a member of. Figure 3b shows how GraphTraverse processes the same query: Step 1: It first obtains all groups. Step 2: For each group, it searches for all its members. Step 3: For each person, it finds all places that he/she visits and checks whether it is located within $Q$.

enclosed with a red rectangle in the figure. Such query should return all possible mappings, including $f_{1}=\{$ (Group, G1), (Person, Bob), (Place, $\left.\left.s_{13}\right)\right\}, f_{2}=\left\{(\right.$ Group, $\mathrm{G} 1)$, (Person, Bob), $\left(\right.$ Place,$\left.\left.s_{14}\right)\right\}$, $f_{3}=\left\{\left(\right.\right.$ Group, G1), (Person, Carol), (Place, $\left.\left.s_{11}\right)\right\}, f_{4}=\{($ Group, G1, $)$, (Person, Carol), (Place, $\left.\left.s_{14}\right)\right\}$. Existing languages, e.g., Cypher [4, 7], GeoSPARQL [8], already allow users to write GraSp queries. Figures 3a and 3b depict how the SpaIndex and GraphTraverse process the query given in Figure 2 .

\section{CONTRIBUTION: RISO-TREE}

In this section, we present the index structure of Riso-Tree and how to construct and maintain such index structure.

\subsection{Index Structure}

Similar to the R-Tree, Riso-Tree is a hierarchical tree structure where each node is represented by a minimum bounding rectangle (MBR). MBR of a node is a rectangle that encloses all its children. However, the method of clustering the spatial objects into different subregions in Riso-Tree differs from that in R-Tree (more details in section 3.2). A key difference between Riso-Tree and R-Tree is that Riso-Tree contains pre-materialized subgraph information, which represents a subset of the graph database, to each index node. A subgraph represents the partial graph elements that are connected to at least one spatial vertex $v$ such that $v$ lies within the MBR of the corresponding tree node. The depth of the subgraph is bounded

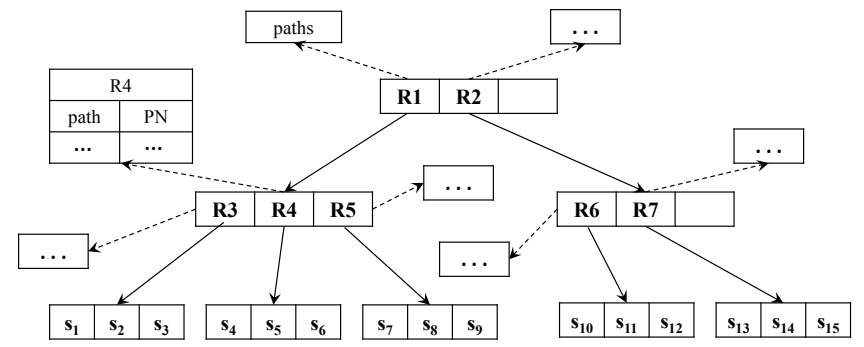

Figure 4: Riso-Tree Memory Structure

by a parameter called $B$, which is tuned by the system user. Every vertex in the materialized subgraph should be within a $B$-hop graph distance from the spatial vertex enclosed by the tree node MBR. Note that $B$ only determines the size of pre-materialized subgraph entries. Hence, its value impacts the pruning power, yet does not affect the query accuracy. All materialized subgraph vertexes in the same Riso-Tree node are packed based on the paths between them and the spatial vertexes lying within the node's MBR.

Given a graph database $G=\{V, E, \varphi, \psi\}$ and $\mathcal{L}$ where $\mathcal{L}$ is the set of all labels in the graph, a label path $p$ can be defined as $p=\mathcal{L}^{*}$. Here $p$ is a sequence of labels generated by Kleene star operation on $\mathcal{L}$. A label path does not include real vertex in the data graph. It represents a path pattern. Use $p[i]$ to denote the label at a specific position. If a real data path $p^{\prime}$ can satisfy $\varphi\left(p^{\prime}[i]\right)=p[i]$ and $\psi\left(p^{\prime}[i], p^{\prime}[i+2]\right)=p[i+1]$ for $i=2 n(n=0,1, \ldots$,$) , which means$ all labels of vertexes and edges on the data path $p^{\prime}$ can be mapped correctly from $p$; hence, we say $p^{\prime}$ can match $p$. For example, in Figure 2, $p=$ Group $-[$ Member $]-$ Person $-[$ Visit $]-$ Place is a label path. The data path $p^{\prime}=G 1-[$ Member $]-B o b-[$ Visit $]-s_{13}$ is a real path in the graph that can match $p$.

Given two vertexes $u^{\prime}$ and $v^{\prime}$, if there exists at least one path $p^{\prime}$ between them that can match $p$, we say that $v^{\prime}$ is reachable from $u^{\prime}$ through path $p$. It is denoted as $u^{\prime} \stackrel{p}{\rightarrow} v^{\prime}$. In the example graph, vertex $B o b$ is reachable from $s_{13}$ through a label path $p=$ Place $-[$ Visit $]-$ Person and it is denoted as $s_{13} \stackrel{p}{\rightarrow}$ Bob. That said, Path Neighbor is defined as follows:

Definition 3.1. Given a vertex $u^{\prime}$ and a label path $p$, Path Neighbor of $u^{\prime}$ with respect to $p\left(P N_{u^{\prime}}^{p}\right)$ is the set of vertexes that satisfy $\left\{v^{\prime} \mid u^{\prime} \stackrel{p}{\rightarrow} v^{\prime}\right\}$.

Definition 3.2. Given a Riso-Tree node $R$ and a label path $p$ in a graph $G=\{V, G\}$, Path Neighbor of $R$ with respect to $p\left(P N_{R}^{p}\right)$ is the set of all vertexes in $G$ that can be reached through the label path $p$ from at least one spatial vertex that lies within $R$.

$$
P N_{R}^{p}=\bigcup_{u \sqsubset R} P N_{u}^{p}
$$

Example. In Figure 2, given a label path $p_{1}$, Path Neighbor of $s_{14}$ with respect to $p_{1}$ is $P N_{s_{4}}^{p_{1}}=\{\mathrm{Bob}, \mathrm{Carol}\}$. Another example, $P N_{R 7}^{p_{2}}$ $=\{G 1\}$ since $G 1$ is the only vertex connected to the spatial vertex enclosed by R7 through the label path $p_{2}$. In the rest of the paper, we refer to a Riso-Tree node by using its MBR.

Basically, graph entries stored in Riso-Tree nodes consist of label paths and Path Neighbors. Figure 4 shows the memory structure 


\begin{tabular}{|c|c|c|c|}
\hline R1 & R3 & R4 & R5 \\
\hline Place - [Visit $]$ - Person & Larry & Joe & Jane \\
\hline Place - [Visit $]$ - Person - $[$ Friend $]$ - Person & Dan, Jane & Alan, Bob & Larry, Carol, Kate \\
\hline Place - $[$ Visit $]$ - Person - $[$ Visit $]$ - Place & $\mathrm{s}_{1}, \mathrm{~s}_{2}, \mathrm{~s}_{3}$ & $\mathrm{~s}_{4}, \mathrm{~s}_{5}, \mathrm{~s}_{6}$ & $\mathrm{~s}_{7}, \mathrm{~s}_{8}, \mathrm{~s}_{9}$ \\
\hline Place - $[$ Visit $]$ - Person - $[$ Member $]$ - Group & $\mathrm{G} 3$ & - & - \\
\hline
\end{tabular}

\begin{tabular}{|c|c|c|}
\hline R2 & R6 & R7 \\
\hline Place - [Visit] - Person & Carol, Kate & Carol, Kate, Bob \\
\hline Place - [Visit] - Person - [Friend $]$ - Person & Jane & Joe, Kate, Jane, Carol \\
\hline Place - $[$ Visit $]$ - Person - $[$ Visit $]$ - Place & $\mathrm{s}_{10}, \mathrm{~s}_{11}, \mathrm{~s}_{14}, \mathrm{~s}_{15}$ & $\mathrm{~s}_{10}, \mathrm{~s}_{11}, \mathrm{~s}_{13}, \mathrm{~s}_{14}, \mathrm{~s}_{15}$ \\
\hline Place - $[$ Visit $]$ - Person - $[$ Member $]$ - Group & G1 & G1 \\
\hline
\end{tabular}

Figure 5: Riso-Tree Path Neighbor Information Example

of the Riso-Tree. Similar to the R-Tree, each Riso-Tree node is represented by an MBR that encloses a set of spatial objects (i.e., spatial vertexes) within its boundary. In addition, each node also stores information about the subgraph connected to the spatial objects enclosed by the node's MBR. Each leaf level node $R$ stores a list of $\left\langle p, P N_{R}^{p}\right\rangle$, where $p$ is a label path and $P N_{R}^{p}$ is the Path Neighbor for such node $R$ with respect to $p$. For non-leaf nodes, only the label paths that exist from the spatial objects within $R$ will be recorded. The length of the considered label paths (i.e., number of hops) is bounded by a predefined parameter $B$. Label Paths longer than $B$ will not be taken into consideration by the Riso-Tree.

Figure 5 depicts an example of a Riso-Tree structure, which corresponds to the graph presented in Figure 2. In this case, $B$ is set to 2 , which means only paths shorter than or equal to 2 are considered. Non-leaf nodes, e.g., R1 and R2, store existing label paths. All their paths are listed in the corresponding tables. On the other hand, Leaf nodes store Path Neighbors. For instance, the subgraph information on R4 is organized by three paths. Path Neighbor with respect to each path is listed in the table as well.

\subsection{Initialization and Maintenance}

The first step of the initialization process is to lay out the Riso-Tree skeleton. Similar to the R-Tree, each newly added spatial object will be inserted into a leaf node and the corresponding MBR will be updated if necessary. When a node is full, it is split into two new nodes with equal number of objects. As opposed to the R-Tree, spatial objects indexed by the Riso-Tree are also a subset of the vertexes in the graph database. Hence, the initialization algorithm takes into account the spatial proximity among entities as well as the proximity of such objects to other elements in the graph.

For a spatial object, its 1-hop neighbors in the graph are utilized to identify its "location" in the graph. For a node in Riso-Tree, which encloses several spatial objects, its 1-hop neighbors are the union of all the 1-hop neighbors of the spatial objects it encloses. Thus, the Graph Similarity (GS) between two spatial objects is measured by using the Jaccard similarity between the two sets as follows:

$$
G S=1-\frac{\left|P N_{a}^{1} \cap P N_{b}^{1}\right|}{\left|P N_{a}^{1}\right|+\left|P N_{b}^{1}\right|-\left|P N_{a}^{1} \cap P N_{b}^{1}\right|}
$$

The graph similarity (value is between 0 and 1 ) between two spatial objects is measured by the number of shared 1-hop neighbors to the total size of their 1-hop neighbors. To make the spatial partitioning graph-aware, Riso-Tree combines graph proximity with spatial

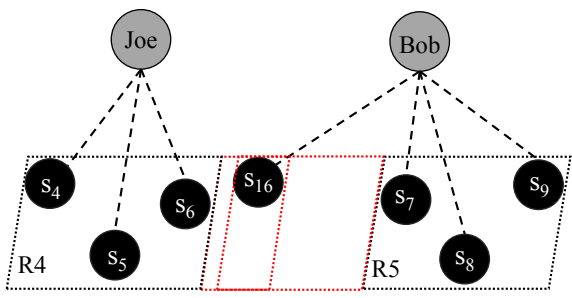

Figure 6: Insert a spatial vertex

proximity. A linear function is exploited to integrate the spatial proximity with graph similarity as follows:

$$
S G D=\alpha \text { Enlarge }+(1-\alpha) G S
$$

Each spatial vertex will be inserted into the leaf node that has the minimum $S G D$. Figure 6 depicts a real example of inserting a new spatial vertex $s_{16}$ into the Riso-Tree. R4 and R5 are two target Riso-Tree nodes for insertion. Their enlargement regions are drawn with red in the figure. If only considering the spatial proximity, $s_{16}$ will be inserted into R4 because the enlargement area of R4 is smaller. But if we consider graph proximity, $P N_{s_{16}}^{1}=\{B o b\}$ and $P N_{R 4}^{1}=\{J o e\}$. So they share no common 1-hop neighbors. $G S\left(s_{16}, R 4\right)=1 . P N_{R 5}^{1}=\{B o b\}$, so $G S\left(s_{16}, R 5\right)=0$. By modifying the value of $\alpha, s_{16}$ might be inserted into $R 5$. In this case, spatial objects within R5 share more common 1-hop neighbors compared to inserting $s_{16}$ into R4.

The reason for adding $G S$ into the final distance function is to reduce the storage overhead. If the two spatial vertices are connected to the same graph vertex $v$ through the same label path and they are inserted into the same leaf node, $v$ only needs to be stored in the shared leaf node. Otherwise, it needs to be stored in two leaf nodes. In the previous example, if $s_{16}$ is added into $R 4, B o b$ needs to be stored in both $R 4$ and $R 5$. By inserting $s_{16}$ into R5, Bob only exist in Path Neighbor of R5.

Node Splitting: When a node is full, it is split into two new nodes with an equal number of objects. Two objects within the full node will be picked as seeds. The two seeds are the first object of each new node. Then the remaining objects will be reassigned to each new node. In an R-Tree, both PickSeeds and DistributeEntry only consider spatial proximity. In the Riso-Tree full node splitting, the algorithm replaces the spatial proximity rule with the new $S G D$ rule mentioned above. The two spatial objects with the maximum $S G D$ will be selected as the seeds. DistributeEntry will distribute each object into the node with smaller $S G D$. To compute $S G D$, each node in the Riso-Tree keeps a 1-hop neighbor set $P N_{R}^{1}$. Each time a spatial vertex is inserted into a node $R, P N_{R}^{1}$ will be updated according to 1-hop neighbors of this spatial vertex.

Once the tree is constructed, the algorithm computes the subgraph entries for each node in the Riso-Tree. The subgraph entries are constructed in a bottom-up manner. Starting from each leaf node in the Riso-Tree, the algorithm computes its Path Neighbors according to the following equation:

$P N_{R}^{p}= \begin{cases}\bigcup_{u \sqsubset R} v \in \operatorname{Adj}(u), \varphi(v)=p[2], \psi(u, v)=p[1] & |p|=1 \\ \bigcup_{u \in P N_{R}^{p^{\prime}}} v \in \operatorname{Adj}(u), \varphi(v)=b, \psi(u, v)=a & p=p^{\prime}-[a]-b .\end{cases}$ 


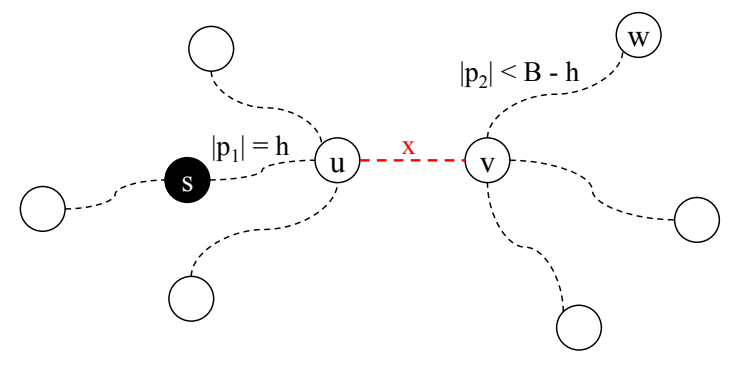

Figure 7: Riso-Tree Maintenance

The algorithm first calculates the node's 1-hop Path Neighbors based on adjacent neighbors of the spatial objects enclosed by this Riso-Tree node. The returned Path Neighbors are divided into different groups according to the different label paths. Once the 1-hop Path Neighbors are constructed, the algorithm computes the 2, 3, ..., B-hop Path Neighbors one after another. Non-leaf nodes are processed from lower to higher levels in the Riso-Tree. Existing paths for each non-leaf node will be accumulated from all its children nodes.

Example. Given the Riso-Tree in Figure $4(B=2)$, the algorithm computes 1-hop Path Neighbor for all leaf nodes, including $R 3$, $R 4, R 5, R 6, R 7$. For instance, Path Neighbor of $\mathrm{R} 7$ with respect to the label path $\left.p_{1} P N_{R 7}^{p}=\bigcup_{s \sqsubset R 7}\left\{u^{\prime} \mid s \stackrel{p_{1}}{\longrightarrow} u^{\prime}\right\}\right)=\{$ Bob, Carol, Kate\}. The algorithm then computes 2-hop Path Neighbor. For instance, Path Neighbor of R7 with respect to a label path $p_{2} P N_{R 7}^{p_{2}}=$ $\cup\left\{u^{\prime} \mid v \rightarrow u^{\prime}, \varphi\left(u^{\prime}\right)=\right.$ Group, $\psi\left(v, u^{\prime}\right)=$ Member $\}=\{\mathrm{G} 1\}$. $v \in P N_{R 7}^{p_{1}}$

Inserting/deleting an edge: Algorithm 1 shows how to update Riso-Tree when an edge is inserted. When an edge of label $x$ between $u$ and $v$ is inserted into the graph, the algorithm first reconstructs Path Neighbors of length from 1 to $B-1$ hops for $u$ and $v$ based on the original graph where the new edge is not considered (Line 2 to 3). The algorithm scans each vertex $w$ in $P N_{u}^{p}$ and $P N_{v}^{p}$ where $|p| \in[1, B-1]$. Foe each spatial vertex $s$, the algorithm updates Path Neighbor information of the leaf node that contains $s$. Besides, all the ancestor nodes of such leaf node will also be updated. Line 4 to 9 show the details of the procedure. Let us use a generic example to demonstrate such a procedure. In Figure 7, a new edge with label $x$ is inserted between $u$ and $v$. There exists one spatial vertex $s$ that is $h$-hop distant from $u$. The Riso-Tree node that contains $s$ is denoted as $R$ (not drawn in the figure). The path from $s$ to $u$ is $p_{1}$. In order to reflect the edge insertion, vertex $v$ should be added into $P N_{R}^{p_{1} x \varphi(v)}$ where $p_{1} x \varphi(v)$ is a label path that concatenates $p_{1}$, $x$ and the label of $v$. Besides that, Path Neighbors of $v, P N_{v}^{p}$ where $|p|<B-h$, will also be added into Path Neighbors of $R$. For a vertex $w$ which is connected from $v$ through path $p_{2}$ and $\left|p_{2}\right|<B-h$, $w$ will be added into $P N_{R}^{p_{1} x p_{2}}$. Besides updating Riso-Tree node $R$, ancestor nodes of $R$ might need to be updated as well. The updating rule is as follows: Existing paths of $R$ 's ancestor nodes need to be updated if the updated path does not exist in $R$ before the insertion. It means that if $p_{1} x p_{2}$ does not exist in $R$ before the updating, then the algorithm adds $p_{1} x p_{2}$ to all the ancestors of $R$.

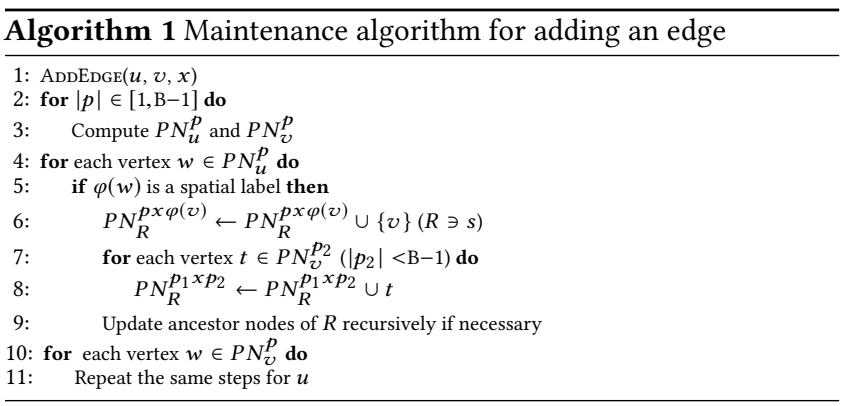

When an edge between $u$ and $v$ is deleted from the graph, the impacted region is similar to inserting an edge. Only the vertexes within B-1 hops from $u$ or $v$ will be impacted. So the first step is still to compute the Path Neighbors within $B-1$ hops from $u$ and $v$. For each spatial vertex $s$ in $P N_{u}^{p}$ and $P N_{v}^{p}$ where $1 \leq|p|<B$, the corresponding Riso-Tree node $R$ that contains $s$ needs to be updated. The way to update the Path Neighbors of $R$ is to recalculate them. Even though such an update is costly, it can be executed lazily without affecting the correctness of future queries.

Inserting/deleting a vertex: Inserting a new vertex with edges attached to it can be decomposed as inserting a solitary vertex and inserting the connected edges. Since the edge insertion has been discussed, we focus on inserting a solitary vertex in this paragraph. If the inserted vertex is not spatial, the algorithm will not change the structure of Riso-Tree. If the vertex is a spatial one, the algorithm will pick up the leaf node to insert that vertex by following the rule of minimizing $S G D$. MBR of the leaf node and the ancestor nodes will be updated accordingly. Similarly, when a non-spatial solitary vertex is deleted from the graph, the algorithm does not need to modify the Riso-Tree. When a solitary spatial vertex $s$ is deleted from a leaf node $R$, the system updates the Riso-Tree in a similar way to the R-Tree. If the number of entries in $R$ is larger than the threshold, the algorithm will just update the MBR of $R$ and its ancestors if necessary. Otherwise, all the entries in $R$ will be reinserted into the Riso-Tree.

Riso-Tree Overhead Analysis. Two components contribute to the Riso-Tree storage cost $\left(S_{\text {riso }}\right)$ : (i) The size of the tree structure denoted as $S_{\text {tree }}$ (ii) Path Neighbor information denoted as $S_{P N}$. Let the fanout of the tree be $f$ and total number of spatial vertexes be $\left|V_{S}\right|$, then the number of levels of Riso-Tree is $\log _{f}\left|V_{S}\right|$. So $S_{\text {tree }}=\sum_{i=0}^{\log _{f}\left|V_{S}\right|} f^{i}$. $S_{P N}$ consists of Path Neighbors and all the existing label paths. Path Neighbors are computed based on $k$-hop neighbors for all spatial vertexes in the network for $0<k \leq B$. Denote the average degree of the graph as $d$. The number of vertexes reachable by all spatial vertexes at specific hop $k$ is $d^{k}\left|V_{S}\right|$. The size of Path Neighbors is $\sum_{k=1}^{B} d^{k}\left|V_{S}\right|$. For each node in the Riso-Tree, the maximum number of label paths it can have is determined by the total number of labels in the graph. Assume that the total number of labels is denoted by $|\mathcal{L}|$. Then, each Riso-Tree node can possess at most $\sum_{k=1}^{B}|\mathcal{L}|^{k}$ paths. In total, the number of Riso-Tree nodes is $\sum_{i=1}^{\log _{f}\left|V_{S}\right|} f^{i}$. So the size of label paths is at most $\left(\sum_{k=1}^{B}|\mathcal{L}|^{k}\right)\left(\sum_{i=1}^{\log _{f}\left|V_{S}\right|} f^{i}\right)$. To sum up, $S_{\text {riso }}$ is as follows: 


$$
\begin{aligned}
S_{\text {riso }} & =\sum_{i=0}^{\log _{f}\left|V_{S}\right|} f^{i}+\sum_{k=1}^{B} d^{k}\left|V_{S}\right|+\left(\sum_{k=1}^{B}|\mathcal{L}|^{k}\right)\left(\sum_{i=1}^{\log _{f}\left|V_{S}\right|} f^{i}\right) \\
& \approx\left(d^{B}+\frac{|\mathcal{L}|^{B}}{f}+1\right)\left|V_{S}\right|
\end{aligned}
$$

The time for building the index $\left(T_{\text {riso }}\right)$ also consists of two parts, the tree skeleton, and the Path Neighbor information, denoted as $T_{\text {tree }}$ and $T_{P N}$ respectively. The tree is constructed by keeping inserting new spatial objects. Each insertion needs to find the leaf node to store the inserted spatial object. It takes $\log _{f}\left|V_{S}\right|$ time. As a result, $T_{\text {tree }}=\left|V_{S}\right| \log _{f}\left|V_{S}\right|$. The construction time also consists of two parts: (1) computing Path Neighbors for leaf nodes and (2) accumulating label paths for non-leaf nodes. The construction needs to access both Riso-Tree nodes and graph vertices. Similarly, the time to compute $P N$ is $\sum_{k=1}^{B} d^{k}\left|V_{S}\right|$ with respect to number of vertices being accessed. To perform such computation, the algorithm also needs to iterate through all the leaf nodes of Riso-Tree, which takes $\frac{\left|V_{S}\right|}{f}$. Then accumulating label paths needs to access the whole Riso-Tree once, which takes $\sum_{i=0}^{\log _{f}\left|V_{S}\right|} f^{i}$. In total, $T_{\text {riso }}$ can be represented as follows:

$$
\begin{aligned}
T_{\text {riso }} & =\left|V_{S}\right| \log _{f}\left|V_{S}\right|+\sum_{k=1}^{B} d^{k}\left|V_{S}\right|+\frac{\left|V_{S}\right|}{f}+\sum_{i=0}^{\log _{f}\left|V_{S}\right|} f^{i} \\
& \approx\left(\log _{f}\left|V_{S}\right|+d^{B}+\frac{2}{f}\right)\left|V_{S}\right|
\end{aligned}
$$

\subsection{Riso-Tree Search Algorithm}

The search algorithm in Riso-Tree takes a rectangular range and a query graph as input such that the input range is attached to a vertex in the query graph. The search algorithm then returns: (1) a set of spatial vertexes that satisfy not only the spatial range but also the query graph and (2) a candidate set for each query vertex that is less than or equal to $B$-hops away from the spatial vertex. Algorithm 2 runs in two main steps as follows:

Step I: Recognize Query Paths (abbr. RecognizePaths) : In the first step, the algorithm finds all the possible label paths in the query graph that are connected to the spatial query vertex belonging to the input range predicate. We call such label path APath. It is formally defined as follows:

Definition 3.3. An APath is a label path $p$ that starts from the spatial query vertex with a range predicate and satisfies $|p| \leq B$, where $B$ is the maximum length of label paths in the Riso-Tree. Such spatial query vertex is called anchor vertex and the end vertex on the other side of the APath is called kite vertex.

For the graph query given in Figure 2, the spatial range predicate is specified on the query vertex of type Place. In that case, Place is the anchor vertex for such spatial predicate. The ReCognizePATHS step detects two APaths, $p_{1}=$ Place $-[$ Visit $]-$ Person and $p_{2}=$ Place - $[$ Visit $]-$ Person- $[$ Member $]-$ Group. The kite vertexes for the two APaths are Person and Group respectively. Basically, the set of APaths of a given anchor vertex depicts the path information of the $B$-hop neighbors of this anchor vertex.
Step II: Generate Reduced Candidate Sets: Once all the APaths are obtained, the algorithm searches the Riso-Tree to generate the reduced candidate sets for the input anchor vertex and its kite vertexes. That way, Riso-Tree can prune: (a) the spatial search space based on subgraph information stored in each Riso-Tree node, and (b) the graph search space based on spatial indexing entries in the Riso-Tree. For a given input $\langle s, Q\rangle$, the algorithm searches the Riso-Tree starting from the root node. For each visited node, the algorithm prunes children nodes whose MBRs are not overlapping with $Q$. The algorithm also prunes the Riso-Tree nodes that do not contain any spatial vertex that can be matched to the query graph. СнескРAтнs (code omitted for brevity) checks the graph constraint to prune unpromising branches in the Riso-Tree. СнескPAтHs takes as input the APaths and the Riso-Tree node $R$ to be checked. If there is any path $p \in A P a t h s$ that does not exist in R.paths, the algorithm will return false, which means that there is no spatial vertex within $R$ that is connected to $p$. The algorithm will return true if and only if $R$ owns all the label paths in APaths.

Consider the query depicted in Figure 2 as an example. If the leaf node R6 is checked whether it can satisfy the graph constraint with the APaths $\left\{p_{1}, p_{2}\right\}$, the function will verify $\left|P N_{R 6}^{p_{1}}\right|$ and $\left|P N_{R 6}^{p_{2}}\right|$. According to the stored Path Neighbor information shown in Figure 5 , both $p_{1}$ and $p_{2}$ are included in R6. Then СНескPATHs will return true in this case. If R4 is checked towards these two APaths, then it will return false because $p_{2}$ is not included by R4.

When the search algorithm reaches the leaf level, the algorithm takes advantage of Path Neighbor stored on this level to generate the reduced candidate sets for the anchor vertex and its kite vertexes. The reduced candidate sets for kite vertexes and anchor vertex are generated in different manners. For a kite vertex, its APath is a constraint on it because this kite vertex needs to be connected to the corresponding query range $Q$ through its APath. The reduced candidate set for a kite vertex with respect to such APath can be computed by using the following equation:

$$
P N_{Q}^{p}=\bigcup P N_{R_{i}}^{p}, R_{i} \cap Q \neq \emptyset \wedge \text { CнескPAтhs }\left(R_{i}, \text { APaths }\right)
$$

where $R_{i}$ is a leaf node of Riso-Tree that overlaps with $Q$ and satisfies the graph constraint. Path Neighbors for $Q$ with respect to $p$ should be the union of Path Neighbors of these leaf nodes.

Example. Consider one of the APaths $p_{1}$ for the query in Figure 2 as an example. The leaf nodes that can satisfy both the spatial and graph constraints are $R 6$ and $R 7$. The reduced candidate set $P N_{Q}^{p_{1}}$ $=P N_{R 6}^{p_{1}} \cup P N_{R 7}^{p_{1}}=\{$ Bob, Carol, Kate $\} \cup\{$ Carol, Kate $\}=\{$ Bob, Carol, Kate\}. So the candidate set for Person is reduced from 8 to 3 .

To obtain the reduced candidate set of an anchor vertex, the algorithm iterates through all the spatial objects that lie within the extents of each leaf node that survives both spatial and graph constraints. For each spatial vertex, the algorithm checks whether it is located within the query rectangle. Since branches that cannot satisfy the graph constraint have been pruned, the size of the candidate set will be smaller than using the traditional range query solution. For the query in Figure 2, the candidate set for $s$ is $\left\{s_{5}, s_{8}\right.$, $\left.s_{10}, s_{11}, s_{13}, s_{14}, s_{15}\right\}$ using the traditional approach. With the help of Riso-Tree, the search can prune R4 and R5 because they do not contain all the APaths. So $s_{5}$ and $s_{8}$ will not be in the candidate set for Place. The final candidate set will be $\left\{s_{10}, s_{11}, s_{13}, s_{14}, s_{15}\right\}$. 


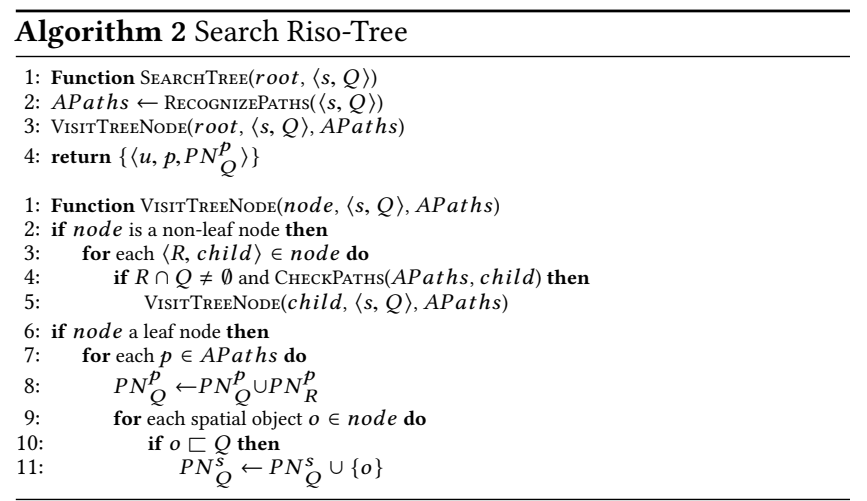

Algorithm 2 shows the pseudo code of Riso-Tree search. The search algorithm takes as input the root node of the Riso-Tree root and spatial range predicate $\langle s, Q\rangle$. The output of the algorithm is the reduced candidate sets for the kite vertexes and anchor vertex. The algorithm first detects all the APaths for the given anchor vertex. Then these APaths are used in the function VisitTreeNode. Inside the VisitTreeNode function, the algorithm follows a recursive way to search the Riso-Tree. Riso-Tree nodes that cannot satisfy the spatial constraint or cannot pass the СнескPAтHs validation will be skipped during the search. The candidate sets for kite vertexes and the anchor vertex are constructed at the leaf level.

\section{QUERY PROCESSING}

In this section, we explain how to harness the Riso-Tree to execute GraSp queries in a graph database system.

\subsection{GraSp-Range}

GraSp-Range query is a graph query with at least one spatial range predicate. Each spatial range predicate is represented as $\langle s, Q\rangle$, where $s$ is a query vertex in the query graph and $Q$ is a spatial rectangle. If a vertex $s^{\prime}$ is mapped from $s$, then it must satisfy not only the query graph but also the range predicate $s^{\prime} \sqsubset Q$. A GraSp-Range query can possess several spatial range predicates, so $S P_{\text {range }}=\{\langle s, Q\rangle\}$ is a set of spatial range predicates. Basically, the solution consists of three main steps, Riso-Tree search, candidate set merge, and query rewrite \& execute.

For each spatial range predicate $\langle s, Q\rangle$ in GraSp-Range, the algorithm first calls the SEARCHTREE algorithm to generate the reduced candidate sets for kite vertexes and the anchor vertex for that predicate. But each candidate set is with respect to each APath. A kite vertex or anchor vertex can exist in different APaths. This can happen when it is connected to one range predicate through different paths or to different range predicates. The APaths that end at the same vertex altogether set constraint on it. So the second step, candidate set merge, will perform the intersection. So its candidate set can be further reduced by the intersection operation. Based on the reduced candidate sets of kite vertexes and anchor vertexes, the algorithm rewrites the original query into a new GraSp-Range $\left(\left\langle G_{q}^{\prime}, S P_{q}^{\prime}\right\rangle\right)$. The new query will share the same graph structure with the original GraSp-Range $\left(G_{q}^{\prime}=G_{q}\right)$, yet with different candidate set. Before the rewriting, the candidate set of each query vertex will be all vertexes with a specific label in the data graph. After rewriting, candidate sets of anchor vertexes and kite vertexes will be reduced to subsets of their original candidate sets. Meanwhile, spatial predicates $S P_{q}^{\prime}$ no longer exist in the GraSp-Range because vertexes in the candidate sets of each anchor vertex definitely satisfy $S P_{q}$. The rewritten version of the query is then executed by the underlying graph database system.

Example. Consider the GraSp-Range in Figure 2 as a running example. There is only one spatial predicate $\langle$ Place, $Q\rangle$ in the query. All the APaths are $\left\{p_{1}, p_{2}\right\}$. There are two kite vertexes, Person and Group and one anchor vertex, Place. The Riso-Tree search will start from the root node. Both $R 1$ and $R 2$ overlap with $Q$ and include both APaths $p_{1}$ and $p_{2}$. Among all the children of R1, R3 cannot satisfy the spatial constraint. R4 and R5 cannot satisfy the graph constraint. So they are all pruned. For node R2, both its children R6 and R7 overlap with $Q$ and pass the СнескPAтнs validation. Then the reduced candidate sets will be constructed based on leaf nodes $R 6$ and R7. The new candidate sets for Person, Group and $\mathrm{Place}$ are constructed as $\{\mathrm{G} 1\},\{\mathrm{Bob}, \mathrm{Carol}, \mathrm{Kate}\}$ and $\left\{s_{10}, s_{11}, s_{13}\right.$, $\left.s_{14}, s_{15}\right\}$ respectively. By calling the SEARCHTREe algorithm, the reduced candidate sets for Group, Person, and Place are constructed as $\{G 1\},\{$ Bob, Carol, Kate $\}$ and $\left\{s_{10}, s_{11}, s_{13}, s_{14}, s_{15}\right\}$ respectively. Since each kite vertex and anchor vertex belong to only one APath, the candidate set merge step will do no computation. Finally, the algorithm rewrites the query based on the new candidate sets. The new query has the same graph structure as the original one in Figure 2 but with reduced search space.

\subsection{GraSp-KNN}

GraSp-KNN integrates a KNN predicate into a graph query. The format of a $\mathrm{KNN}$ predicate is $\langle s, l o c, K\rangle$, where $s$ is the spatial query vertex in the graph and $l o c$ is the input searched location. The query will return top- $K$ closest spatial vertexes from location $l o c$ that can satisfy the query graph. The SpaIndex strategy can be utilized to execute a GraSp-KNN query by extending the classic KNN query processing algorithm, as follows: It first finds the next closest spatial object by using the incremental KNN algorithm. For each spatial object, the algorithm then checks whether it can satisfy the query graph. If it can satisfy the graph constraint, add it to the result set. Otherwise, fetch the next closest spatial vertex and perform the same operation until $K$ satisfying objects are found. Such an approach is still inefficient since so many spatial objects that cannot satisfy the graph constraint will still be accessed. Furthermore, the validation of the graph query constraint(s) is very time-consuming. To remedy that, our proposed GraSp-KNN query processing algorithm exploits the Riso-Tree to prune unnecessary tree branches as early as possible to reduce the amount of graph constraint validation.

The algorithm takes as input a query graph $G_{q}$ and a KNN predicate $\langle s, l o c, K\rangle$. The first step is still to recognize the APaths with respect to the anchor vertex $s$. A priority queue $q$ is used and the sorting key is the distance between an object (either a Riso-Tree node or a spatial object) and the query location loc. The root node root $_{s}$ of the Riso-Tree is accessed first. If all the APaths are included in the paths connected to roots, then roots is enqueued to $q$. Each time, the head element $e$ will be dequeued. Basically, $e$ can be a non-leaf node, a leaf node or a spatial object. If $e$ is a non-leaf node, 


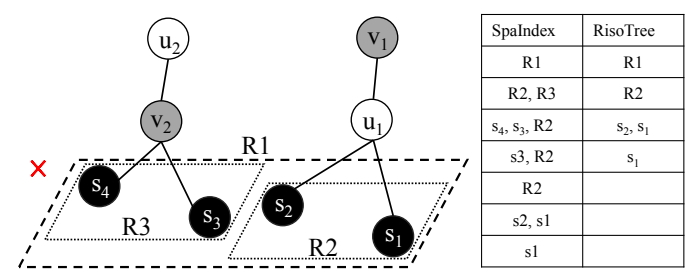

Figure 8: Query Example for GraSp-KNN

for each child of $e$, it is checked towards all the APaths by using СнескPAтhs function. If the function returns true, such child node will be enqueued along with its distance to loc. If $e$ is a leaf node, each child of $e$ which is a spatial object will be directly enqueued. For the final case that $e$ is a spatial object, the algorithm will rewrite the graph query that maps $s$ to $e$, and execute the query. If there is at least map, $e$ is added to the result. Such procedure continues until $K$ objects are found or $q$ is empty.

Example. Figure 8 shows an example of GraSp-KNN. The query location loc is drawn with a red cross in the figure. The query is $(v-u-s,\langle s, l o c\rangle)$. Assume that $K$ is equal to 1 . So the aim of such query is to find the closest $s$ vertex from loc that can satisfy $v-u-s$. The table in Figure 8 depicts the elements in the queue for SpaIndex and Riso-Tree approach during the query precessing. SpaIndex first accesses all the spatial objects within R3, which are $s_{3}$ and $s_{4}$ and performs graph search on them. However, $s_{3}$ and $s_{4}$ fail the search. Then R2 is dequeued and its children are enqueued. Finally, $s_{2}$ is found as the final result. But, Riso-Tree can avoid accessing spatial objects within R3. The reason is that existing paths for R3 are $v-u$ and $v$ so that does not include the APaths in the query.

\subsection{GraSp-Join}

GraSp-Join query is a graph query with at least one spatial join predicate that joins two sets of spatial vertexes in the graph database. A spatial join predicate is commonly used in geospatial analytics. It can be any spatial relationship between spatial objects, such as overlap, enclosed by, within distance, etc. Without loss of generality, we present our work based on the "Within distance', spatial join operator, which means that two spatial objects need to be within a given distance from each other. The format of the spatial join predicate is $S P_{q}=\langle s, t, d i s t\rangle$, where $s$ and $t$ are the spatial query vertexes included in the distance join predicate and dist is the distance value. An example query is to find all the pairs of friend vertexes in a social graph who live within $1 \mathrm{~km}$ from each other.

Similar to performing spatial join by using R-Tree, the algorithm (pseudocode omitted for brevity) keeps pairs of nodes in the RisoTree in a queue $q$. It starts by pushing the root nodes of Riso-Tree into $q$. Each time, a pair of Riso-Tree nodes $\left\langle\right.$ node $_{a}$, node $\left._{b}\right\rangle$ is dequeued. For each child of node $e_{a}$ and node ${ }_{b}$, the algorithm checks both distance join predicate and graph constraint by using CHECKPATHs function. The pairs that can pass the validation are enqueued. If the nodes are leaf nodes, each pair of spatial vertexes will be checked against the spatial join predicate. The pairs that can satisfy the predicate will be added to the candidate set. The candidate set for the two anchor vertexes in each join predicate is much smaller as compared to the SpaIndex approach. This is because our algorithm

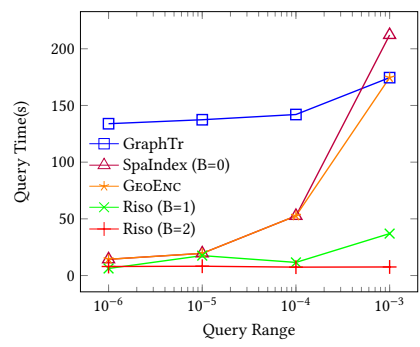

(a) Gowalla

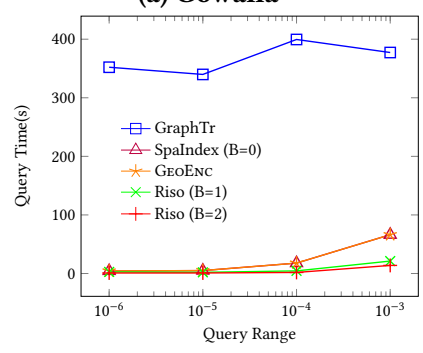

(c) Foursquare

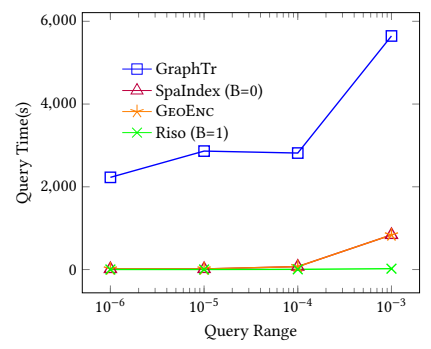

(b) WikiData

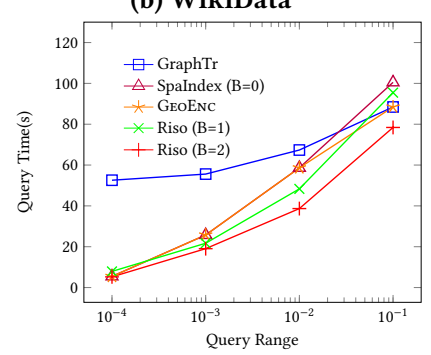

(d) Yelp
Figure 9: GraSp-Range query response time

filters out spatial vertexes that cannot satisfy the graph constraint without searching the graph. Once the candidate set for all anchor vertexes are generated, our approach rewrites the original query into a set of graph queries for each pair of spatial objects to search for the subgraphs that can satisfy the query graph.

\section{EXPERIMENTS}

In this section, we experimentally evaluate the proposed approach. We compare Riso-Tree to the SpaIndex, GraphTraverse and GeoEnC approaches. GEoENC is the spatial encoding approach implemented in [10]. GEoENC was implemented for RDF stores, but we adopted the encoding approach to property graphs in Neo4j. For Riso-Tree, we set the value of $B$ to 0,1 (default), 2 and they are denoted as SpaIndex $(B=0)$, Riso $(B=1)$ and Riso $(B=2)$, respectively. A prototype of all approaches is implemented in $\mathrm{Neo} 4 \mathrm{j}$ - a real open source graph database management system. The source code for evaluating query response time is implemented in Java and compiled with java-7openjdk-amd64. All experiments are run on a computer with an 3.60 GHz CPU, 32GB RAM running Ubuntu 16.04 Linux.

We evaluate the compared approaches using the following metrics: (i) Initialization time: the average time (in Seconds) each approach takes to construct all indexes and/or auxiliary data structures necessary to answer a GraSp, (ii) Storage overhead: the disk space (in Bytes) used to store the index and auxiliary data structures, (iii) Query response time: the average time the GDBMS takes to execute a GraSp query. We use four real datasets: (1) WikiData: A Knowledge graph with $\approx 47$ Million vertexes $(\approx 6$ Million vertexes are spatial) and $\approx 330$ Million edges. (2) Gowalla: geosocial graph dataset extracted from SNAP datasets ${ }^{1}$ with $1477 \mathrm{~K}$ vertexes, 5878K edges, and $1280 \mathrm{~K}$ spatial vertexes. (3) Foursquare: a geosocial graph dataset [16] with $3296 \mathrm{~K}$ vertexes, $1143 \mathrm{~K}$ spatial vertexes,

\footnotetext{
${ }^{1}$ https://snap.stanford.edu/data/loc-gowalla.html
} 
and 18723K edges. (4) YELP ${ }^{2}$ : an urban knowledge graph dataset (extracted from the Yelp Challenge dataset) with $629 \mathrm{k}$ vertexes $(12 \%$ are spatial) and $8503 \mathrm{~K}$ edges.

Query Generation. The query graphs are generated by exploiting the subgraph generator used in the existing paper [13]. A core vertex is randomly picked up from the original data graph and a subgraph around this core vertex is generated by random walk. It can ensure that the query graph will definitely have subgraph matchings in the data graph. In the GraSp-Range, the spatial range selectivity is evaluated by the number of spatial objects in the query range to the total number of spatial objects in the graph.

Impact of Spatial Selectivity on GraSp-Range: Graph queries used for such evaluation have the size of 10, 3, 10 and 5 in four datasets. The lengths of the longest simple paths in each query graph are 5, 3, 5 and 3 . For each query graph, we test 50 rectangles on each spatial selectivity. The average time is recorded. Figure 9 shows the query time of different approaches. For each dataset, we evaluate different spatial selectivities. The query time of GraphTraverse does not change dramatically when spatial selectivity varies. The reason is that GraphTraverse always follows the same graph search strategy and the range constraint is always checked after the spatial vertexes are visited. SpaIndex $(B=0)$ can outperform GraphTraverse in all the datasets except for the highest spatial selectivity case in Gowalla and Yelp datasets. It is because R-Tree can reduce the size of the candidate set for the spatial query vertex. Such a reduction factor is determined by the spatial selectivity. Hence, the query time of SpaIndex $(B=0)$ increases when the spatial predicate is less selective because more spatial vertexes lie within the query rectangle. For Gowalla, the number of spatial vertexes (86\% of all vertexes) is too high. The query time of SpaIndex $(B=0)$ increases fast and exceeds the query time of GraphTraverse when selectivity is $10^{-3}$. That happens because accessing the R-Tree becomes an overhead when the number of spatial objects within the query range is high. In all datasets, Riso-Tree approach can outperform both SpaIndex $(B=0)$ and GraphTraverse because the candidate sets for query vertexes within $B$-hop range of the spatial predicate can be reduced by orders of magnitude by using Riso-Tree. Specifically, Riso $(B=2)$ can achieve even better performance than Riso $(B=1)$ because Riso $(B=2)$ can reduce the candidates of kite vertexes up to 2-hop neighbors of the anchor vertex while Riso $(B=1)$ is 1-hop. The query response time of Riso-Tree tends to increase when there are more spatial vertexes in the query range since the effect of reducing the size of candidate sets diminishes in that case.

Impact of Query Graph Size on GraSp-KNN: Figure 10 depicts the query time of GraSp-KNN by varying the query graph size (number of vertexes in the query graph) in Foursquare dataset. The size of query graphs varies from 3 to 9 . Each query is run for 3 times. For both SpaIndex and Riso $(B=2)$ method, it takes longer processing time when the size of the query graph increases. Recall that for each visited spatial vertex, the algorithm will perform graph search to validate whether it can satisfy the query graph. It requires a longer time to search the graph for a larger-sized query graph. But Riso $(\mathrm{B}=2)$ can always outperform SpaIndex because Riso $(B=2)$ performs much less query graph validations than SpaIndex. Thanks to the pre-stored Path Neighbor information on each node

\footnotetext{
${ }^{2}$ https://www.yelp.com/dataset_challenge
}

in the Riso-Tree, Riso $(B=2)$ method can prune many unpromising branches in the spatial index. The pruned spatial vertexes will not appear in the graph constraint validation phase, which means the graph search does not happen from these pruned spatial vertexes.

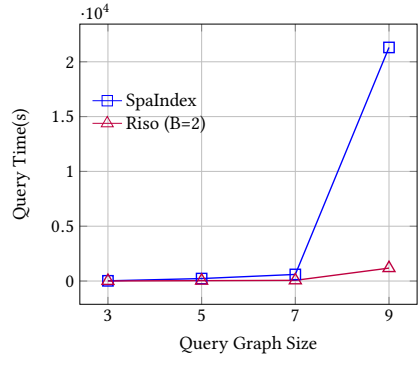

(a) varying query graph size

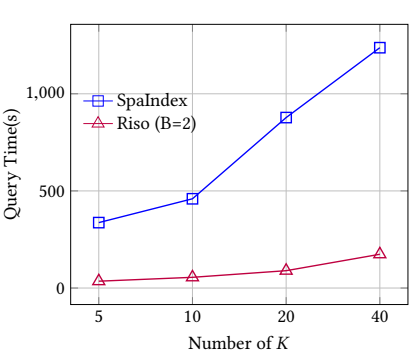

(b) varying $K$
Figure 10: GraSp-KNN on Foursquare

Impact of K on GraSp-KNN: Figure 10b shows the query time of SpaIndex and Riso $(\mathrm{B}=2)$ by varying the value of $K$ in the query. The query graph used in this figure has 5 vertexes. Query time of SpaIndex and Riso $(B=2)$ increases as $K$ increases from 5 to 40. Because the two approaches incrementally find the top $K$ closest spatial vertexes that can satisfy the query graph, larger $K$ means that they need to find more spatial objects, which takes longer time. When comparing Riso $(B=2)$ with SpaIndex, Riso $(B=2)$ can always outperform SpaIndex up to $10 x$ times better. Riso $(B=2)$ can take advantage of the Riso-Tree to prune some unnecessary branches in the Riso-Tree. So Riso $(B=2)$ checks much fewer spatial vertexes than SpaIndex approach.

GraSp-Join Performance: To analyze the influence of spatial join distance, we vary its value in the GraSp-Join. The query graph has 5 vertexes. Each query is run for 3 times. Figure 11a shows the query time for GraSp-Join in Foursquare and WikiData datasets under different join distances. When the join distance increases, the query time of both SpaIndex and Riso $(B=2)$ increase because there are more pairs of spatial vertexes that are within the join distance. Riso $(\mathrm{B}=2)$ can achieve up to 10 times better performance than SpaIndex approach. Since Riso $(B=2)$ has much fewer spatial vertexes to process than SpaIndex during the graph search phase, Riso $(B=2)$ takes less time than SpaIndex.

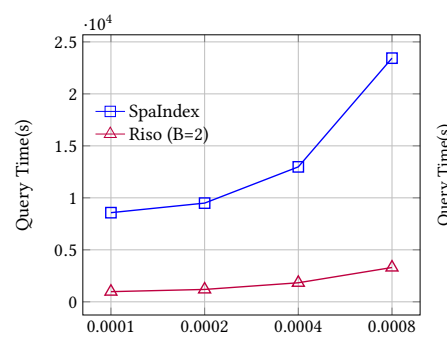

(a) Foursquare

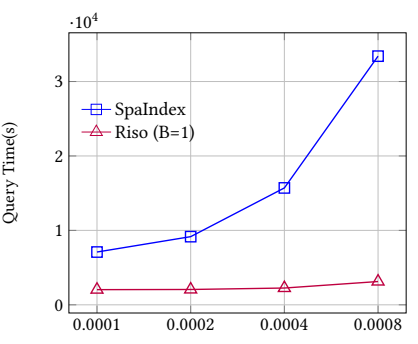

(b) WikiData
Figure 11: GraSp-Join by varying join distance

Studying the indexing overhead. Table 1 shows the indexing overhead of Riso-Tree with different $B$ s. When $B=0$, Riso-Tree has 
Table 1: Indexing Overhead

\begin{tabular}{|c|c|c|}
\hline Gowalla & Initialization Time $(\mathrm{s})$ & Storage Overhead (MB) \\
\hline SpaIndex $(\mathrm{B}=0)$ & 27.07 & 24.79 \\
\hline Riso $(\mathrm{B}=1)$ & 28.67 & 32.14 \\
\hline Riso $(\mathrm{B}=2)$ & 64.73 & 1145.21 \\
\hline \hline Wikidata & Initialization Time $(\mathrm{s})$ & Storage Overhead (MB) \\
\hline SpaIndex (B=0) & 151.70 & 111.42 \\
\hline Riso (B=1) & 232.08 & 221.43 \\
\hline \hline Foursquare & Initialization Time (s) & Storage Overhead (MB) \\
\hline SpaIndex $(\mathrm{B}=0)$ & 28.90 & 22.10 \\
\hline Riso $(\mathrm{B}=1)$ & 30.12 & 25.41 \\
\hline Riso $(\mathrm{B}=2)$ & 32.53 & 71.24 \\
\hline \hline Yelp & Initialization Time $(\mathrm{s})$ & Storage Overhead (MB) \\
\hline SpaIndex $(\mathrm{B}=0)$ & 4.15 & 1.52 \\
\hline Riso $(\mathrm{B}=1)$ & 4.71 & 10.88 \\
\hline Riso $(\mathrm{B}=2)$ & 21.50 & 618.01 \\
\hline
\end{tabular}

exactly the same structure with Riso-Tree. Index overhead of Riso $(\mathrm{B}=1)$ is higher than SpaIndex $(\mathrm{B}=0)$ because it needs to handle the 1hop Path Neighbor in the Riso-Tree. Riso $(B=1)$ is just slightly worse than SpaIndex $(B=0)$ for all the datasets upon the construction time and index size. But Riso $(B=2)$ is far worse than Riso $(B=1)$ for most of the datasets because the number of 2-hop Path Neighbors is far more than 1-hop Path Neighbors. The reason is that the number of neighbors in a graph will increase exponentially with respect to the number of hops. Construction time of the largest dataset WikiData even exceeds 1 hour and the index size is larger than 200GB. So we do not consider the $B$ value of 2 in the WikiData dataset.
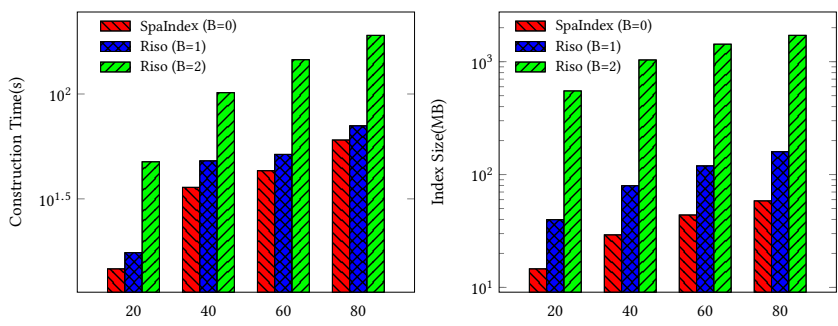

(a) Init. Time (Spatial Ratios)

(b) Index Size (Spatial Ratios)

Figure 12: Indexing Overhead

Figures $12 \mathrm{a}$ and $12 \mathrm{~b}$ show the indexing overhead for a synthetic dataset that simulates various spatial to non-spatial vertexes ratios. The synthetic dataset consists of $3775 \mathrm{~K}$ vertexes and $16519 \mathrm{~K}$ edges. We randomly pick up $20 \%, 40 \%, 60 \%$ and $80 \%$ vertexes as spatial objects. Their location is randomly selected in a $[0,1000] \times[0,1000]$ $2 \mathrm{D}$ space. When the ratio of spatial vertexes increases, both the initialization time and storage size increase. For SpaIndex $(B=0)$, the size of the tree skeleton is determined by the number of spatial objects. More spatial vertexes will lead to more nodes at each level. Hence, it takes more time and space to construct and store Path Neighbor. That also explains why the overhead of Riso $(B=1)$ and Riso $(B=2)$ is higher when the number of spatial vertexes increases.

\section{CONCLUSION}

In this paper, we propose Riso-Tree, a hierarchical spatial index that stores precomputed sub-graph entries in each of its nodes. The Riso-Tree search algorithm leverages both the spatial indexing entries and the sub-graph entries to prune the search space before the query is even executed by the graph database system. That makes Riso-Tree a generic index that can efficiently evaluate general purpose spatial predicates in graph queries. The paper proves through empirical analysis the superiority of the Riso-Tree over classic query processing approaches adopted by state-of-the-art graph database engines. In fact, the experiments show that our proposed graph query processing algorithms built based upon the Riso-Tree structure can achieve up to two orders of magnitude better query execution performance than existing state-of-the-art approaches. We believe Riso-Tree will spark future research directions in hybrid graph/spatial stores, especially with the ubiquity of geo-location and graph data in many applications. We also plan to apply Riso-Tree to applications like spatial entity resolution, discovering related spatial entities, and finding factual answers in geographic knowledge graphs. We will also investigate how GraSp queries run in a distributed data processing system.

\section{REFERENCES}

[1] Armenatzoglou, N., Papadopoulos, S., and Papadias, D. A general framework for geo-social query processing. PVLDB 6, 10 (2013), 913-924.

[2] Bakalov, P., Hoel, E. G., AND Kim, S. A network model for the utility domain. In SIGSPATIAL/GIS (2017), ACM, pp. 32:1-32:10.

[3] Beckmann, N., Kriegel, H., Schneider, R., And Seeger, B. The $r^{*}$-tree: An efficient and robust access method for points and rectangles. In SIGMOD (1990), pp. 322-331.

[4] Cypher language. https://neo4j.com/developer/cypher-query-language/.

[5] Doytsher, Y., Galon, B., AND Kanza, Y. Querying geo-social data by bridging spatial networks and social networks. In ACM LBSN (2010), ACM, pp. 39-46.

[6] Doytsher, Y., Galon, B., AND KanZA, Y. Querying socio-spatial networks on the world-wide web. In $W W W$ (2012), ACM, pp. 329-332.

[7] Francis, N., Green, A., Guagliardo, P., Libkin, L., LindaAker, T., Marsault, V., Plantikow, S., Rydberg, M., Selmer, P., and Taylor, A. Cypher: An evolving query language for property graphs. In SIGMOD (2018), ACM.

[8] Geosparql. http://www.opengeospatial.org/standards/geosparql.

[9] Guttman, A. R-Trees: A Dynamic Index Structure For Spatial Searching. In SIGMOD (1984).

[10] Liagouris, J., Mamoulis, N., Bouros, P., and Terrovitis, M. An effective encoding scheme for spatial RDF data. PVLDB 7, 12 (2014), 1271-1282.

[11] Mouratidis, K., Li, J., TANG, Y., And Mamoulis, N. Joint search by social and spatial proximity. TKDE 27, 3 (2015), 781-793.

[12] Neo4j graph database. https://neo4j.com/.

[13] REN, X., AND WANG, J. Multi-query optimization for subgraph isomorphism search. Proc. VLDB Endow. 10, 3 (Nov. 2016), 121-132.

[14] SAMEt, H. Foundations of Multidimensional and Metric Data Structures. Morgan Kaufmann, 2006.

[15] Sarwat, M., Bao, J., Chow, C., Levandoski, J. J., Magdy, A., And Mokbel, M. F. Context awareness in mobile systems. In Data Management in Pervasive Systems. 2015, pp. 257-287.

[16] Sarwat, M., Levandoski, J. J., Eldawy, A., and Mokbel, M. F. LARS*: An Efficient and Scalable Location-Aware Recommender System. TKDE 26, 6 (2014), $1384-1399$.

[17] Shi, J., Mamoulis, N., Wu, D., And Cheung, D. W. Density-based place clustering in geo-social networks. In SIGMOD (2014), ACM, pp. 99-110.

[18] Sun, W., Fokoue, A., Srinivas, K., Kementsietsidis, A., Hu, G., And Xie, G. T. Sqlgraph: An efficient relational-based property graph store. In SIGMOD (2015), pp. 1887-1901.

[19] Sun, Y., Pasumarthy, N., And Sarwat, M. On Evaluating Social Proximity-Aware Spatial Range Queries. In MDM (2017).

[20] Titan distributed graph database. http://titan.thinkaurelius.com/.

[21] VRANDEČrć, D., AND KRÖTzsch, M. Wikidata: A free collaborative knowledgebase. Commun. ACM 57, 10 (Sept. 2014), 78-85. 